\title{
Extraordinary Properties of Epsilon-Near-Zero and Low-Index Chalcogenide Metamaterials
}

\author{
D. Piccinotti' ${ }^{1}$, B. Gholipour ${ }^{1,2}$, J. Yao ${ }^{2}$, K. F. Macdonald ${ }^{1}$, B. E. Hayden ${ }^{2}$ and N. I. Zheludevi, 3 \\ 1. Optoelectronics Research Centre and Centre for Photonic Metamaterials, University of Southampton, Southampton, SO17 1BJ, UK \\ 2. Department of Chemistry, University of Southampton, Southampton, SO17 1BJ, UK \\ 3. Centre for Disruptive Photonic Technologies, TPI, SPMS, Nanyang Technological University, Singapore 637371
}

\begin{abstract}
Laminar flow' transmission, suppression of plasmonic powerflow vortices, and absorption decreasing with increasing Joule losses can be observed in metamaterials combining plasmonic nanostructures with chalcogenide inclusions exhibiting epsilon-near-zero and subunitary refractive index behaviors.

OCIS codes: (160.3918) Metamaterials; (160.6000) Semiconductor materials
\end{abstract}

Chalcogenide semiconductor epsilon-near-zero (ENZ) and low-index media give rise to a variety of intriguing and counterintuitive behaviors in nanostructured plasmonic metamaterials. For example, 'turbulence' in the near-field flow of light around sub-wavelength apertures in a plasmonic metal screen can be suppressed by filling them with an ENZ medium (Fig. 1), increasing transmission over a broad spectral range. Unexpectedly though, transmission is not necessarily enhanced at the epsilon-nearest-zero wavelength, and a reduction of losses in the chalcogenide can increase, rather than decrease, metasurface absorption.

It is well-appreciated that nano-structuring of plasmonic and high-index, low-loss dielectric materials can provide a remarkable level of control over optical properties, and underlying light-matter interactions at the sub-wavelength scale. In this work we analyze some of the still more fascinating opportunities offered by structuring materials with highly-dispersive ultraviolet to near-infrared optical properties between those of ideally plasmonic (e.g. noble metal) and transparent dielectric media. Chalcogenide semiconductors are good examples - they can exhibit positive, negative and zero values of the real part of relative permittivity $\left(\varepsilon_{l}\right)$, and refractive index values $\leq 1$. Our analysis is based in the first instance upon ellipsometrically measured material parameters (i.e. including realistic losses) for vapor deposited antimony telluride $\left(\mathrm{Sb}_{2} \mathrm{Te}_{3}\right)$.

We find that $\mathrm{Sb}_{2} \mathrm{Te}_{3}$ inclusions can serve as conduits for the 'laminar flow' of light through deeply sub-wavelength slots in a plasmonic metal screen (Fig. 1): around empty slots, 'whirlpools' of optical energy are formed,

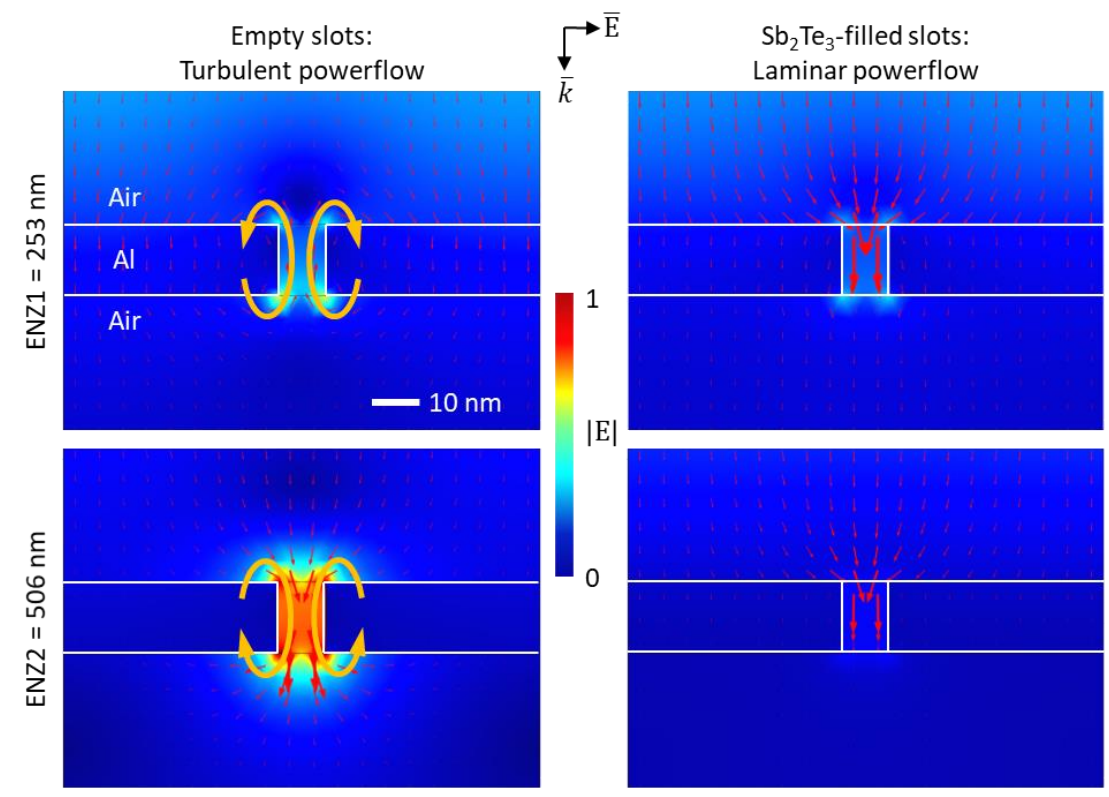

Fig. 1: Laminar vs. turbulent light flow through an Al nano-slot metasurface. Cross-sectional distributions of electric field, overlaid with arrows showing time-averaged powerflow, for $\mathrm{Al}$ metasurfaces (of the geometry shown in Fig. 2a) with empty (left) and $\mathrm{Sb}_{2} \mathrm{Te}_{3-}$ filled (right) slots at the chalcogenide's two $\varepsilon_{l}$ zero-crossing wavelengths, ENZ1, $2=253$ and $506 \mathrm{~nm}$ (upper and lower rows respectively). 
(a)

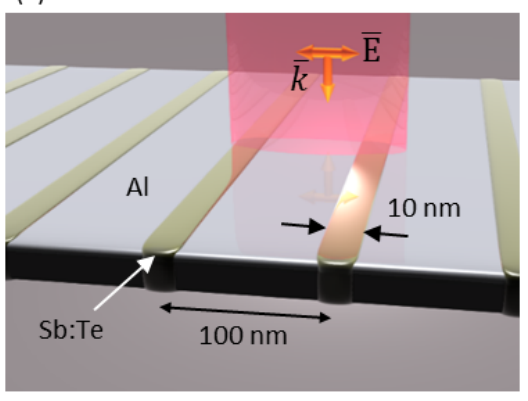

(b)

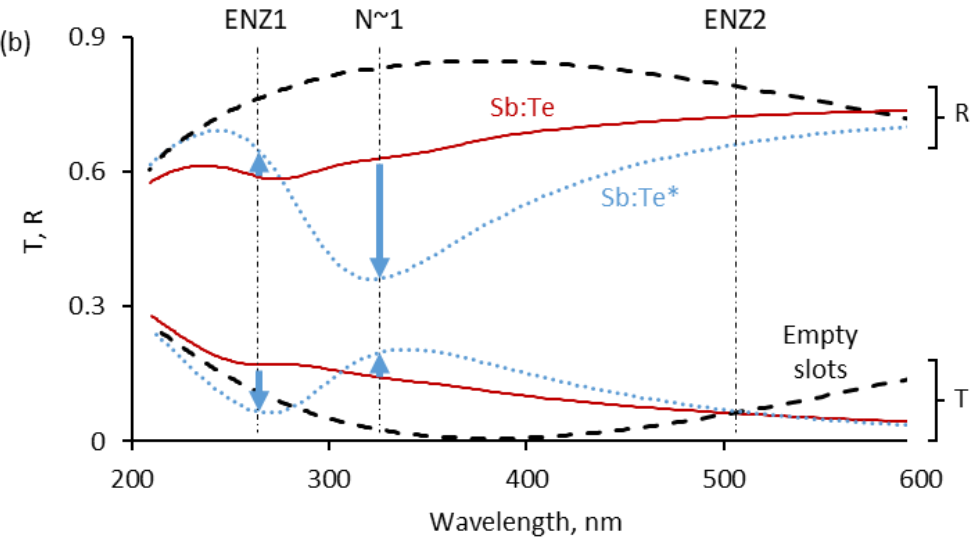

Fig. 2: Metamaterial structure and optical properties at special dispersion points (ENZ and N 1). (a) Schematic illustration of the numerically simulated metasurface, comprising a periodic array of linear slots (width $<<$ illumination wavelength $\lambda$, period $<\lambda$ ) in a 15 $\mathrm{nm}$ thick Al film. (b) Numerically simulated reflection $R$ and transmission $T$ spectra for the metasurface with empty slots [black dashed lines], $\mathrm{Sb}_{2} \mathrm{Te}_{3}$-filled slots [solid red], and slots filled with a hypothetical medium Sb:Te* [dotted blue] having losses - imaginary part of permittivity $\varepsilon_{2}$ - reduced by a factor of 8 relative to $\mathrm{Sb}_{2} \mathrm{Te}_{3}$ at $\lambda_{1}$. Blue arrows denote key changes arising from the reduction of losses in the chalcogenide.

characteristically rotating (as around plasmonic nanoparticles) in opposite directions at wavelengths either side of a plasmonic resonance; $\mathrm{Sb}_{2} \mathrm{Te}_{3}$ inclusions funnel light through the nanoscale apertures, suppressing the turbulent powerflow whirlpools and associated local field enhancement, resulting in a broadband enhancement of transmission (Fig 2b). From analytical studies of ideal ENZ media, one might anticipate that this effect would be most pronounced at the low-loss $\varepsilon_{l}=0$ wavelength ENZ1, but that is not the case. Indeed, an analysis using a Kramers-Kronig-compliant oscillator model for the dispersion of antimony telluride's complex permittivity reveals that with reduced losses, transmission may actually be suppressed at ENZ1, while absorption is increased where the magnitude of refractive index $|N|=\sqrt{\left(n^{2}+k^{2}\right)} \sim 1$, i.e. matched to that of the surroundings.

With optical and electronic properties that can be controlled by composition and (amorphous/crystalline) phase state, chalcogenides represent a flexible, CMOS-compatible material platform for UV-to-NIR nanophotonics. Using high-throughput physical vapor deposition and characterization techniques we explore the ENZ, plasmonic and lowindex characteristics of Bi:Sb:Te alloys: Thin film samples in which composition varies continuously over a substrate are produced by co-deposition of constituent elements, each with a defined density gradient. In this way, optical, electronic, structural and thermal properties can be rapidly mapped to composition. It is found for example (Fig. 3), that the VIS/NIR $\varepsilon_{1}$ zero-crossing wavelength ENZ2 is a strong function of composition, being tunable from $\sim 500$ out to nearly $1700 \mathrm{~nm}$.
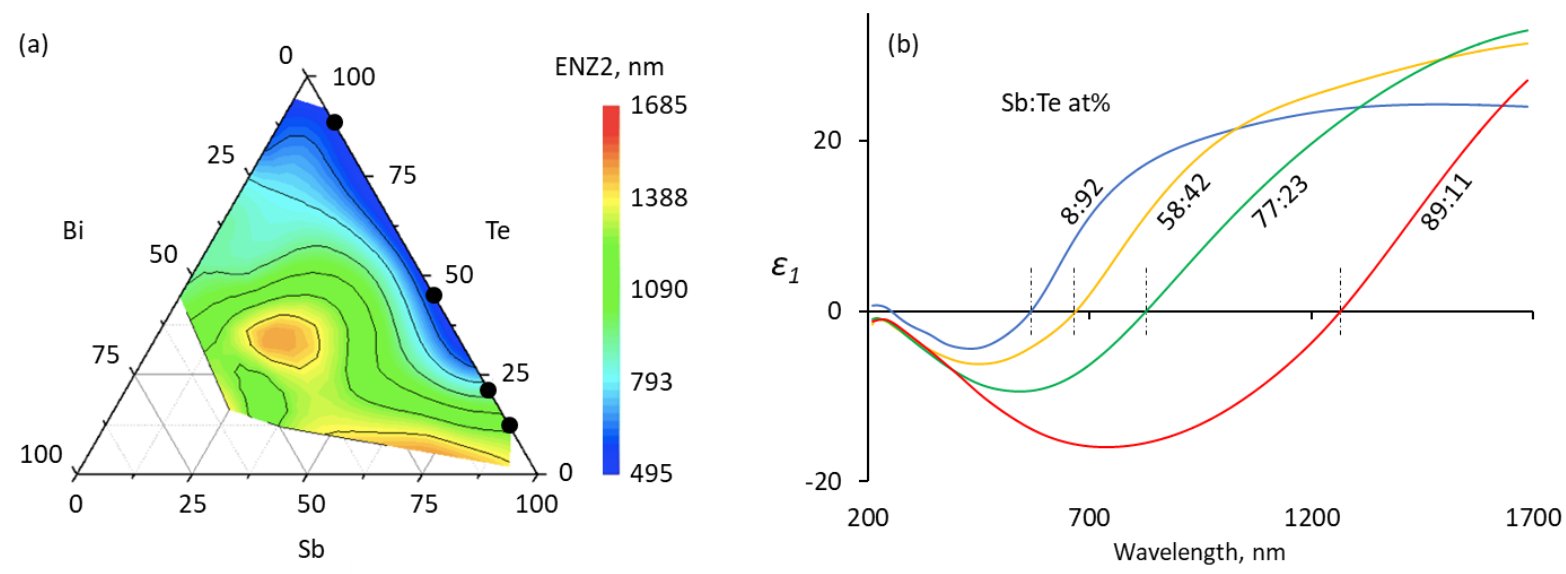

Fig. 2: Compositional tuning of chalcogenide optical properties. (a) Color map of the VIS to NIR wavelength ENZ2 at which the real part of relative permittivity $\varepsilon_{l}=0$ as a function of alloy composition for the Bi:Sb:Te ternary system. (b) Spectral dispersion of $\varepsilon_{l}$ for a selection of binary $\mathrm{Sb}$ :Te compositions [at\% ratios as labelled, corresponding to the marked point $0 \% \mathrm{Bi}$ axis in panel (a)]. 